\title{
FABLE REPRESENTATION IN FNOK AND DNOK FORMALISMS USING THE NOK CONCEPTUAL FRAMEWORK
}

\author{
Marina Rauker Koch, Martina Ašenbrener Katić \& Mile Pavlić
}
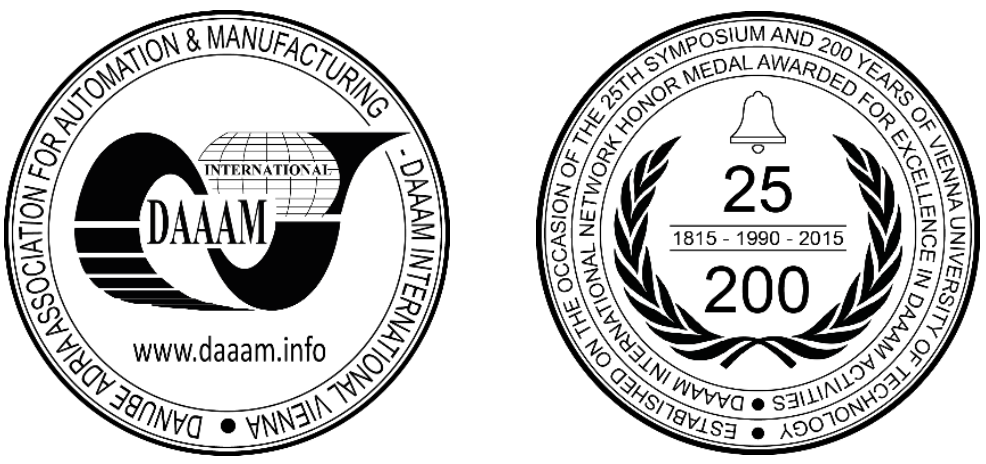

This Publication has to be referred as: Rauker Koch, M[arina]; Asenbrener Katic, M[artina] \& Pavlic, M[ile] (2017). Fable Representation in FNOK and DNOK Formalisms Using the NOK Conceptual Framework, Proceedings of the 28th DAAAM International Symposium, pp.0439-0445, B. Katalinic (Ed.), Published by DAAAM International, ISBN 9783-902734-11-2, ISSN 1726-9679, Vienna, Austria

DOI: $10.2507 / 28$ th.daaam.proceedings.061

\begin{abstract}
The conceptual framework NOK (Node of Knowledge) consists of the NOK method, and DNOK and FNOK formalisms. Formalism DNOK enables a graphical representation and organization of knowledge in the form of a diagram as network nodes, and FNOK formalism represents knowledge in a textual form, which is suitable for entry into a relational database. This paper describes the representation of a text in DNOK form and formalized FNOK form, on the example of Aesop's fable "The Goose with the Golden Egg". The sentences from the fable consist of at least two actions, that is, they contain two verbs, and the paper shows that DNOK and FNOK formalisms can also be applied to such complex sentences.
\end{abstract}

Keywords: Node of knowledge (NOK); FNOK; DNOK; Knowledge Representation; Aesop

\section{Introduction}

Knowledge is a term that cannot be precisely determined and defined [1]. To convert knowledge into a form that can be coded using a computer, many methods have been developed applicable to different types of knowledge [2], [3]. The conceptual framework Node of Knowledge (NOK) is intended to represent sentences written in natural language [4].

In this paper, the conceptual framework NOK was applied to sentences from Aesop's fable "The Goose with the Golden Egg" [5], which are shown graphically in the form of DNOK (Diagram Nodes Of Knowledge) and in the formalized FNOK form [6]. Our aim is to show that the NOK conceptual framework, i.e. the NOK method, and the DNOK and FNOK formalisms can be applied to the so-called "complex sentences", i.e. sentences containing two or more verbs (multiple related actions). Examples of such complex sentences can be seen in this fable. The ultimate goal is to investigate data in the relational database, and it is therefore important to transform complex sentences into a form suitable for entering into a relational database. By using the conceptual framework NOK, we can transform complex sentences into a FNOK form that can be entered into a relational database, while DNOK serves as a more transparent and simplified representation, as shown in this paper. The second chapter gives an overview of relevant research in which the conceptual framework NOK was developed. 
The third chapter describes the reasons for conducting this research, and the fourth chapter describes the applied methodology. The fifth chapter describes the application of DNOK and FNOK formalisms to sentences from the fable "The Goose with the Golden Egg". The sixth chapter describes the results, conclusions and possible problems of applying NOK to complex sentences, as well as plans for future research.

\section{An Overview of Relevant Research}

Knowledge Representation (KR) is one of the subtypes of artificial intelligence [7] and deals with methods of formal representation of knowledge in a computer. A lot of research is concerned with finding a method for knowledge representation [2], [8], [9], [10], [11], [12].

The NOK method belongs to a group of semantic networks that represent knowledge in a form of a graph. Its goal is to display textual knowledge as a knowledge network [4]. The formalization of the NOK method is presented in [6], [13]. This method analyses natural human language, sentences, words and their meanings, as well as related strings of words that form more complex phrases [14]. The NOK method translates knowledge into the model and then conducts a knowledge search in the model. It is then able to answer questions related to that knowledge.

Some of the problems of working with natural language are also homonyms and synonyms, and the model to resolve the problems is described in [15].

\section{Research Motivation}

The conceptual framework Nodes of Knowledge (NOK) consists of methods for modelling sentences written in a natural language. One fable in two languages translated by two different authors is shown in [16]. A knowledge network is created for these translations using the NOK method. For each of the fable versions, a diagram is created which contains all sentences from the fable. Then, the Croatian version of DNOK and the English version of DNOK are compared.

It is shown that the NOK method is applicable to modelling of sentences written in different natural human languages. Given the freedom of translation, some parts of the model remain similar, but there are some differences in the same story, in the same sentences.

This paper aims at graphically representing each sentence from the English version of the fable "The Goose with the Golden Egg" [5]in DNOK and formalized FNOK form. This will also show that complex sentences, with multiple related actions, can be represented in DNOK and FNOK forms. The resulting sentences are then suitable for entry into the relational database, which will be the subject of future research.

\section{Methodology}

The authors of the paper use the "one-way" NOK method in which the link between the nodes is realized by one node asking the question and the other node answering the question through the relationship in the model graphically represented in DNOK form. Fig. 1. shows an example of DNOK for the simple sentence "Ana is happy".

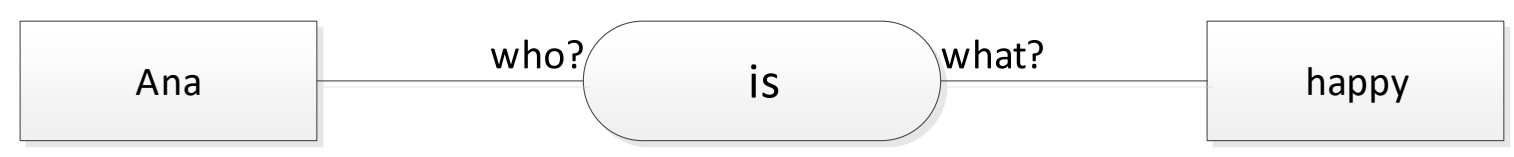

Fig. 1. DNOK for the sentence „Ana is happy.“

FNOK for this sentence is:

$$
\text { is("who?" Ana, "what?” happy) }
$$

Complex human knowledge can be recorded in the form of a diagram that differs from the human language in such a way that one term exists only once in the DNOK but is linked as many times as necessary (unlimited number of links) [17], [14]. Node is the smallest element of knowledge that cannot be divided, and every new term or new meaning is a new node and it is indivisible [4]. Thus nodes can contain named persons, things, events, actions, ideas. The graphical representation of the basic concepts in the NOK method is given in Table 1.

Links connect nodes into a knowledge network. Links are represented by lines and have roles in the form of questions that connect two neighbouring nodes[18].

For this method, a formalized sentence representation was developed, i.e. the FNOK formalism [6], [13] is used to transform the sentences. The procedure for transforming sentences into FNOK form is given in [6], and it enables sentences with semantic link between nodes, using wh-questions, to be displayed textually [19]. 


\begin{tabular}{|c|l|}
\hline \multicolumn{1}{|c|}{ Symbol } & \multicolumn{1}{c|}{ Meaning } \\
\hline & $\begin{array}{l}\text { NODE - a part of knowledge in reality or } \\
\text { thoughts }\end{array}$ \\
\hline & PROCESS NODE - action, event, activity \\
\hline Role (question) & $\begin{array}{l}\text { LINK - "one-way" link and a question with the } \\
\text { link - linking nodes }\end{array}$ \\
\hline
\end{tabular}

Table 1. NOK symbols applied to modelling

\section{Research Results}

\subsection{Translating into DNOK and FNOK}

For the example of textual knowledge, the Aesop's fable "The Goose with the Golden Egg" [5] is used and separated into sentences as follows:

1. One day a countryman going to the nest of his Goose found there an egg all yellow and glittering.

2. When he took it up it was as heavy as lead and he was going to throw it away, because he thought a trick had been played upon him.

3. But he took it home on second thoughts, and soon found to his delight that it was an egg of pure gold.

4. Every morning the same thing occurred, and he soon became rich by selling his eggs.

5. As he grew rich he grew greedy; and thinking to get at once all the gold the Goose could give, he killed it and opened it only to find nothing.

Each sentence is translated into DNOK and FNOK forms separately. The first sentence was taken and each word in it was analysed and categorised according to word type. Verbs were separated from all the other word types. Each sentence in the fable contains more than one verb, so the sentences are complex. In simple sentences with only one verb, the verb is placed in the first position.

Here it was necessary to determine the exact semantic affiliation and the hierarchy between the verbs, i.e. the sequence of actions, so the first verb from the hierarchy is placed in the first position. If two actions, two verbs, in the sentence are mutually connected by conjunctions, then we place the conjunction in the first position, like in the second, third, fourth and fifth sentence. We link other verbs and words using questions with verbs on the higher level they belong to.

\subsection{Fable Representation in DNOK}

Each word in the sentence, as the smallest unit of knowledge, represents one node. Verbs, including conjunctions in complex sentences which link two clauses, are represented as process nodes in the ellipsis symbol. All the other nodes are represented in rectangular symbols. Semantically linked nodes are connected using links that contain a role, i.e. the question to which the other node in the representation gives the answer. In Fig. 2, the second node egg answers the question found what?.

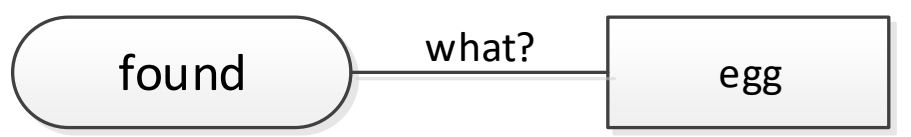

Fig. 1. Link between two nodes

For a more detailed description of the procedure, the fourth sentence is selected from the fable because, besides being complex, it contains three verbs, two of which are linked by a conjunction. The fourth sentence reads: Every morning the same thing occurred, and he soon became rich by selling his eggs. Sentence analysis resulted in these verbs: occurred, became and selling. Two simple clauses are connected into one complex sentence and interconnected using the conjunction and.

In this case, the conjunction and is observed as a process node and placed at the top of the hierarchy under which we then place verbs occurred and became. The verb selling is placed at a new level below became because it comes after became. At the next level, we put words related to verbs (e.g., morning, thing) and we continue to build the hierarchy depending on the interrelationship between the words (Fig. 4). 


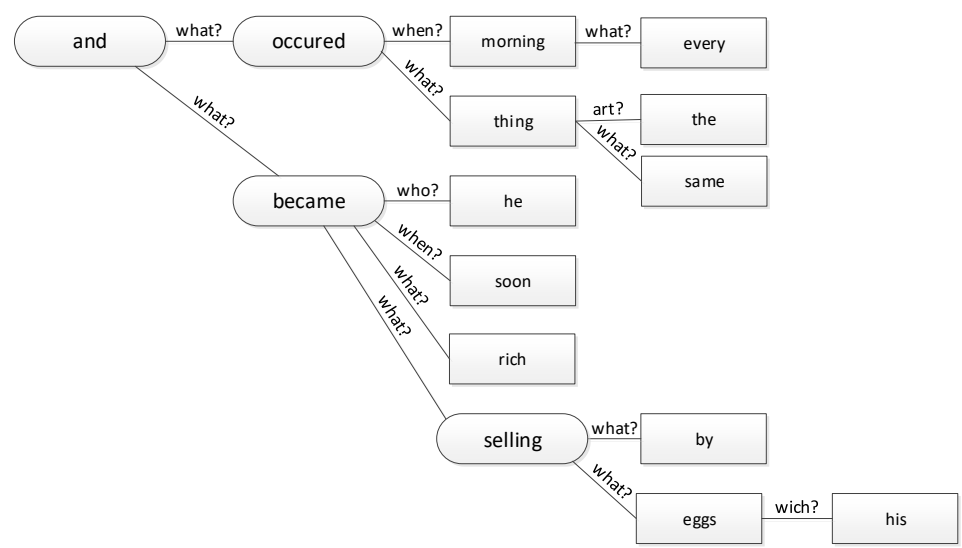

Fig. 2. DNOK of the fourth sentence

For the remaining sentences, the corresponding graphical representations in the form of DNOK are shown in Fig. 4 to Fig. 7.

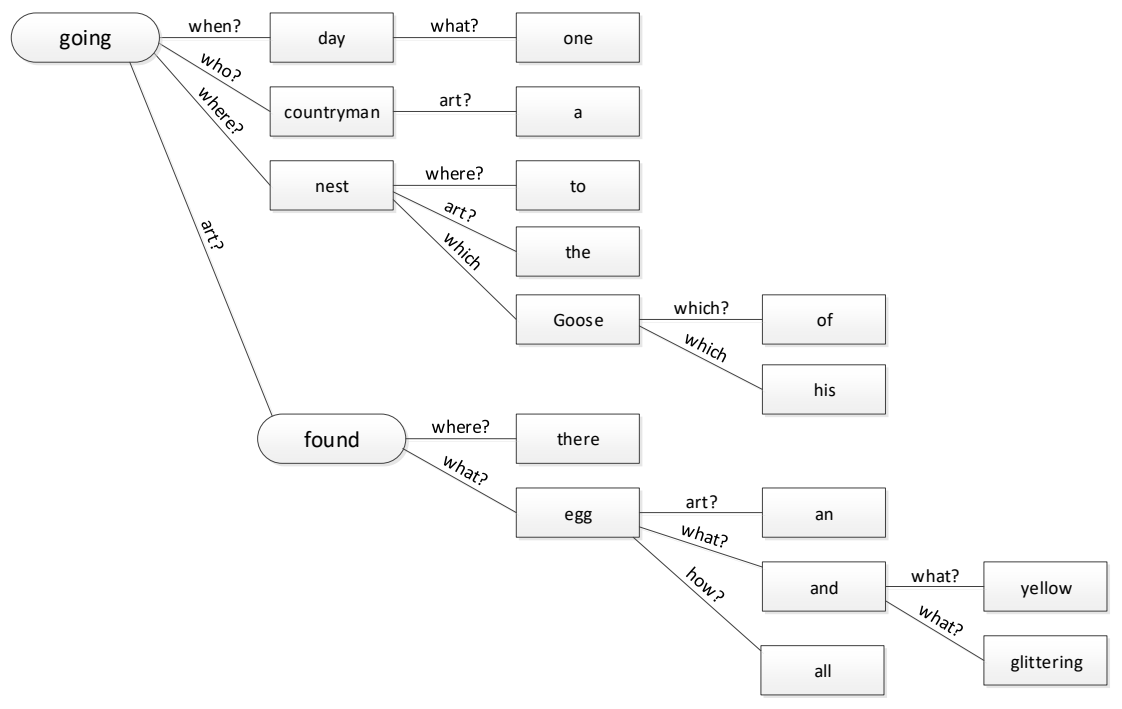

Fig. 3. DNOK of the first sentence

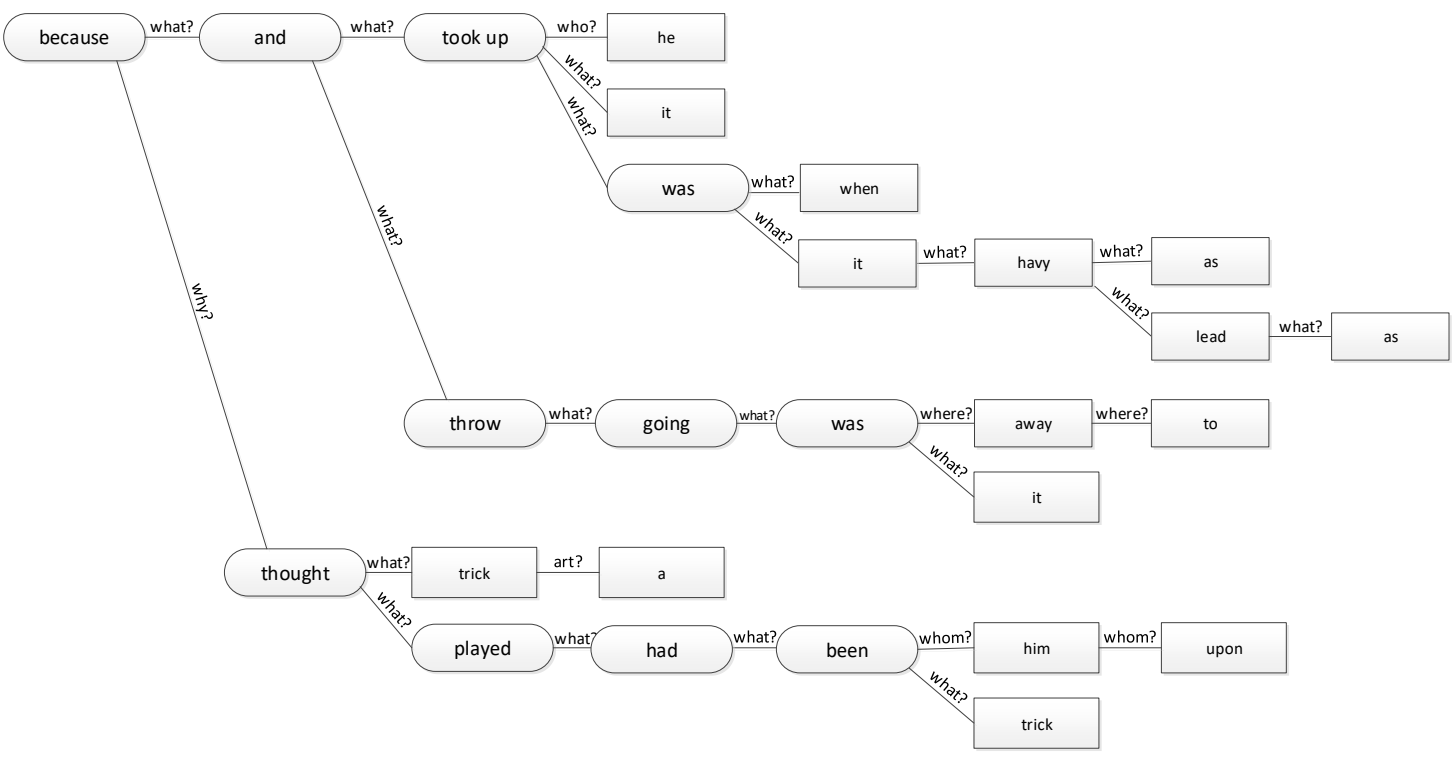

Fig. 4. DNOK of the second sentence 


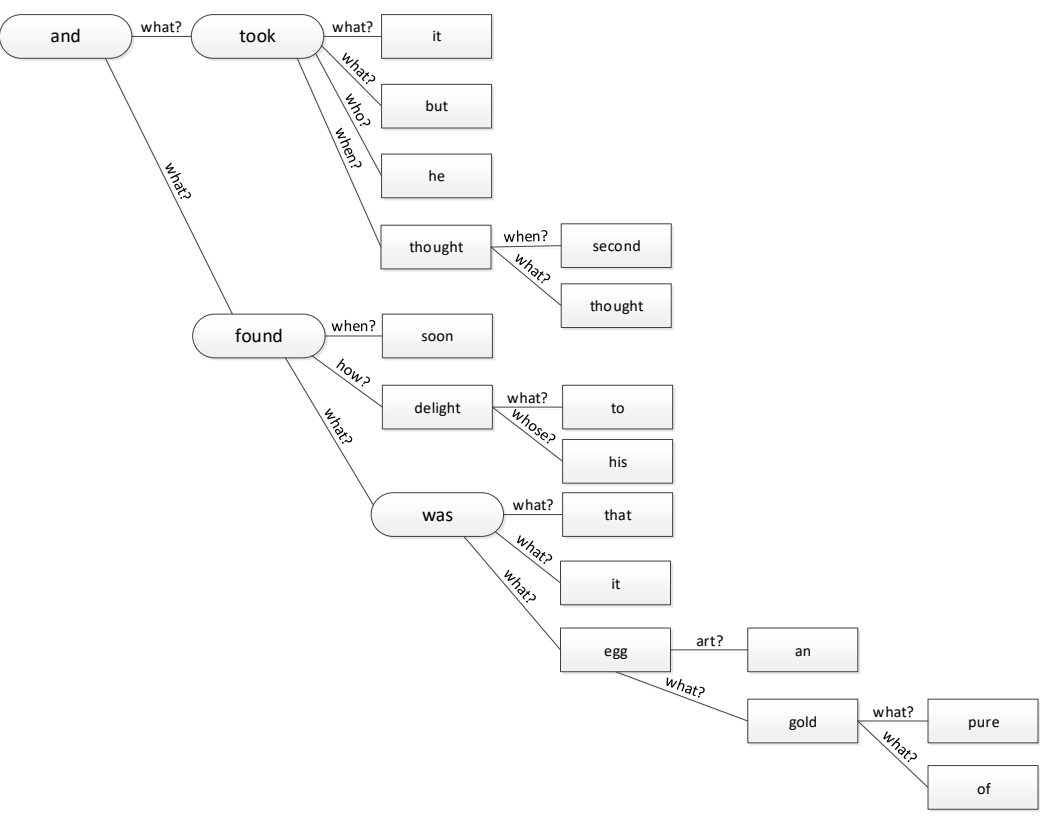

Fig. 5. DNOK of the third sentence

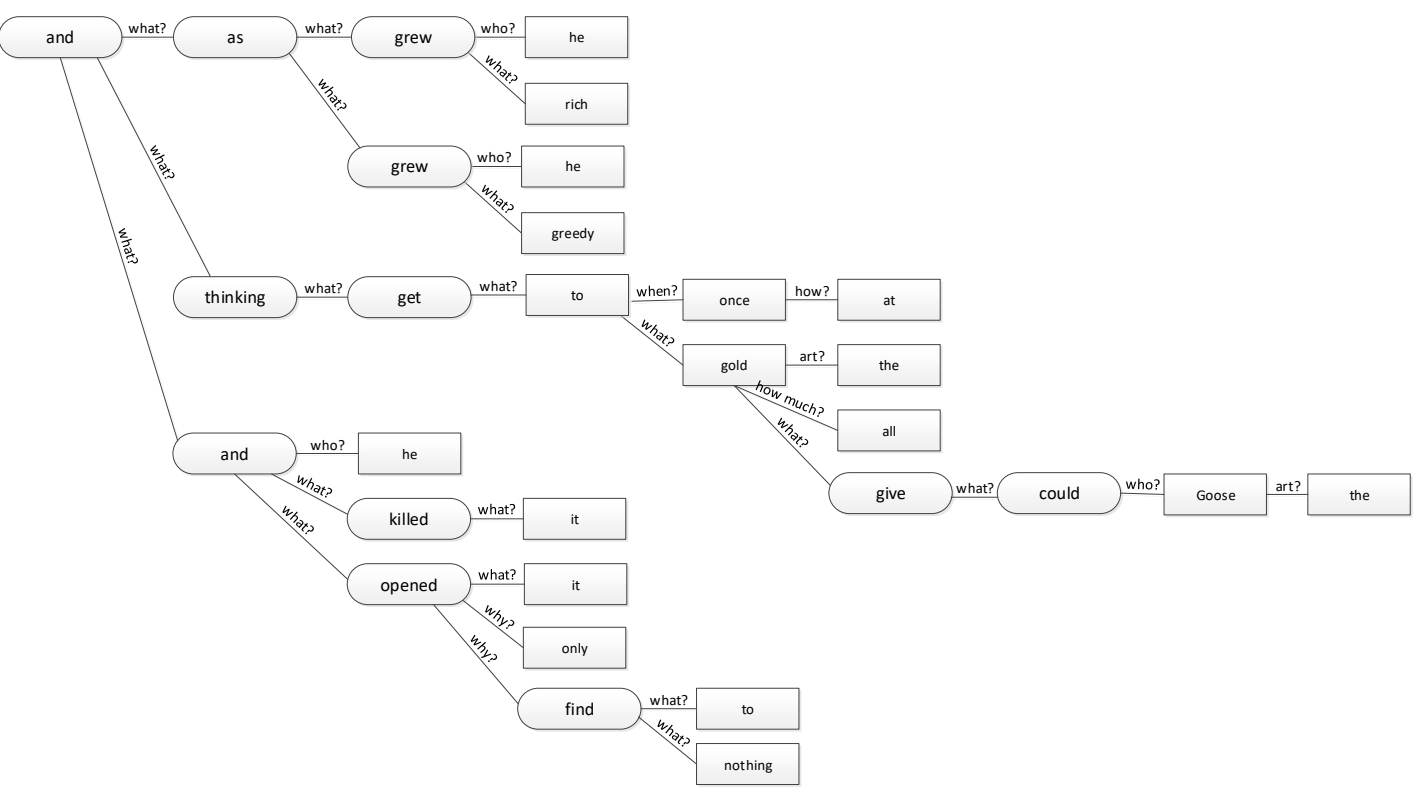

Fig. 6. DNOK of the fifth sentence

\subsection{FNOK Representation of Fable Sentences}

In order to prepare the sentences from the fable to be entered into the relational database, they need to be represented in a formalized FNOK form. When creating FNOK forms, a hierarchy visible in DNOK is followed, by opening parentheses for each new level, while the words from sentences with corresponding questions on the same level are separated by a comma.

The fable sentences in FNOK form:

1. going (,when?“ day (,what?“ one), ,who?“ countryman (,,art?“ a), ,where?“" nest (,,where?“ to, ,,art?“ the, „which?“ Goose ("which?” of, "which?” his)), "what?” found (“where?” there, "what?” egg (“art?” an, "how?” all, "what?” and ("what?" yellow , "what?" glittering))))

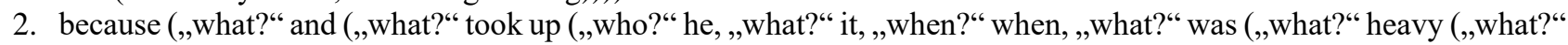
as, ,what?“ lead (,what?“ as)))), ,,what?“ throw (,what?“ was (,what?“" going (,where?“ away (,,where?“ to), ,what?“ it)))), ,why?" thought (,,what?" trick (,,art?“a), ,what?“ played (,what?“ had (,,what?“ been (,,whom?“ him (,,whom?“ upon), ,what?“ trick)))) 
3. and (,what?“ took (,what?“ it, ,what?“ but, „,who?“ he, ,what?“ thought ( “when?” on, ,when?“ second)), ,what?“ found (,when?“ soon, „,how?“ delight (,,what?“ to, ,whose?“ his), ,what?“ was (,what?“ that, ,,what?“ it, „,what?“ egg (,,art? an, „what?“" gold (,,what?“ of, „what?“" pure))))))

4. and (,what?“ occurred (,when?“ morning (,what?“" every), ,what?“ thing (,,art?“ the, ,what?" same), ,what?" became (,who?“ he, ,when?“ soon, ,what?“ rich, ,what?“ selling (,what?“ by, ,what?” eggs (,which?“ his)))))

5. and (“what?” as (“what?” grew (,who?“" he, ,what?“ rich), ,what?“" grew (,,who?" he, ,what?“ greedy)), “what?” thinking (,what?“" get (,what?“ to, ,when?“ once (,,how?“ at), ,what?“ gold (,,art?“ the, ,how much?“" all, ,what?“ give (,what?" could (,,who?" Goose (,,art?" the))))))), "what?” and (“who?” he, "what?” killed (“what?” it), "what?” opened (,what?“ it, ,why?“ only, „why?“ find (,what?“ to, “what?" nothing))))

\section{Conclusion}

This paper presents the application of the conceptual framework NOK and DNOK formalism, which is used to present the sentences graphically in the form of a diagram, and FNOK formalism, where the sentences are presented in a formalized form. So far, these formalisms have only been applied to simple sentences (sentences containing a verb in the present tense, i.e. a sentence represents one action). This paper went a step further; the research was extended to complex sentences and it was shown that complex sentences can be represented in DNOK and FNOK forms. The conceptual framework NOK was applied to sentences from the fable "The Goose with the Golden Egg", which are complex and each of them consists of at least two interdependent actions (verbs). By applying these methods, the interdependence is not lost and the semantics of the sentence is preserved, because every word is linked through a role (question) to the word it is related to. Complex sentences often contain conjunctions that link the two actions, and in that case their role is different from merely connecting two words. Therefore, they are placed at the top of the hierarchy. In simple sentences the words at the top of the hierarchy are verbs, and in the graphical representation they are represented as process nodes. Likewise, the conjunction at the top of the hierarchy is also represented as a process node. Types of conjunctions and their role in the sentence will be the subject of future research.

This research has shown that the conceptual framework NOK can be applied to complex sentences, because, in order to transform the fable into a relational database, it is necessary to represent it in a formalized form (FNOK), as well as graphically in the form of a NOK diagram (DNOK) so that the relationships between the nodes are more clearly presented. Transforming complex sentences into the relational database will be the subject of future research.

Future research will also concentrate on: comma analysis, different tenses, interrogative and reflexive pronouns, developing models and algorithms that will enable querying the relational database, the problem of synonyms and homonyms and etc.

\section{Acknowledgments}

The research has been conducted under the project "Extending the information system development methodology with artificial intelligence methods" (reference number 13.13.1.2.01.) supported by University of Rijeka (Croatia).

\section{References}

[1] Brachman, R. \& Levesque, H. (2004). Knowledge representation and reasoning. The Morgan Kaufmann Series in Artificial Intelligence, Morgan Kaufmann.

[2] Harmelen, F., Lifschitz, V. \& Porter, B. (2008). Handbook of Knowledge Representation. Elsevier, Oxford.

[3] Russell, S. \& Norvig, P. (2010). Artificial Intelligence: A Modern Approach, Third edition. Prentice Hall.

[4] Pavlić, M., Jakupović, A. \& Meštrović, A. (2013). Nodes of knowledge method for knowledge representation. Informatologia, 46, pp. 206-214.

[5] Aesop. The Goose with the golden egg, Available from: www.bartleby.com/17/1/57.html, Accessed: 2017-06-10

[6] Jakupović, A., Pavlić, M. \& Dovedan Han, Z. (2014). Formalisation method for the text expressed knowledge. Expert Systems with Applications, 41, pp. 5308-5322. https://doi.org/10.1016/j.eswa.2014.03.006

[7] Morgenstern, L. \& Thomason, R.H. (2000). Teaching Knowledge representation: Challenges and Proposals. KR2000,

[8] Sowa, J.F. (2000). Knowledge representation: logical, philosophical, and computational foundations. Pacific Grove: Brooks/Cole.

[9] Chein, M. \& Mugnier, M.-L. (2009). Graph-based Knowledge Representation, Computational Foundations of Conceptual Graphs. Springer.

[10] King, T.H. \& Helbig, H. (2009). Knowledge Representation and the Semantics of Natural Language. Natural Language Engineering, 15, 310.

[11] Sowa, J.F. (2014). Principles of semantic networks: Explorations in the representation of knowledge. Morgan Kaufmann.

[12] Kamp, H. \& Reyle, U. (2013). From discourse to logic: Introduction to modeltheoretic semantics of natural language, formal logic and discourse representation theory. Springer Science \& Business Media. 
[13] Pavlić, M., Dovedan Han, Z. \& Jakupović, A. (2015). Question answering with a conceptual framework for knowledge-based system development "Node of Knowledge." Expert Systems with Applications, 42, 5264-5286. https://doi.org/10.1016/j.eswa.2015.02.024

[14] Asenbrener Katic, M., Pavlic, M. \& Candrlic, S. (2015). The Representation of Database Content and Structure Using the NOK Method. 25th Daaam International Symposium on Intelligent Manufacturing and Automation 2014, Procedia Engineering, 100, pp. 1075-1081. https://doi.org/10.1016/j.proeng.2015.01.469

[15] Rauker Koch, M., Pavlic, M. \& Asenbrener Katic, M. (2015). Homonyms and Synonyms in NOK Method. 25th Daaam International Symposium on Intelligent Manufacturing and Automation 2014, Procedia Engineering, 100, pp. 1055-1061. https://doi.org/10.1016/j.proeng.2015.01.466

[16] Rauker Koch, M., Pavlić, M. \& Jakupović, A. (2014). Application of the NOK method in sentence modelling. Proceedings of the 37th Internation Convention MIPRO 2014, Croatian Society for Information and Communication Technology, Electronics - MIPRO. pp. 1426-1431.

[17] Pavlic, M., Dovedan Han, Z., Jakupovic, A., Asenbrener Katic, M. \& Candrlic, S. (2017). Adjective representation with the method Nodes of Knowledge. 2017 40th International Convention on Information and Communication Technology, Electronics and Microelectronics (MIPRO), IEEE. https://doi.org/10.23919/MIPRO.2017.7973610

[18] Pavlić, M., Mestrović, A. \& Jakupović, A. (2013). Graph-based formalisms for knowledge representation. Proceedings of the 17th World Multi- Conference on Systemic Cybernetics and Informatics (WMSCI 2013), Orlando. pp. 200-204.

[19] Asenbrener Katic, M., Candrlic, S. \& Pavlic, M. (2017). Comparison of Two Versions of Formalization Method for Text Expressed Knowledge. Beyond Databases, Architectures and Structures (BDAS) 2017, CCIS, pp. 55-66. https://doi.org/10.1007/978-3-319-58274-0_5 